\title{
Deciding Conjugacy in Thompson's Group F in Linear Time
}

Nabil Hossain, Robert W. McGrail, and James Belk

The Laboratory for Algebraic and Symbolic Computation

Reem Kayden Center for Science and Computation

Bard College

Annandle-on-Hudson, New York 12528

\author{
Francesco Matucci \\ Département de Mathématiques \\ Faculté des Sciences d'Orsay \\ Université Paris-Sud 11 \\ Bâtiment 425, Bureau 21 F-91405 Orsay Cedex, France
}
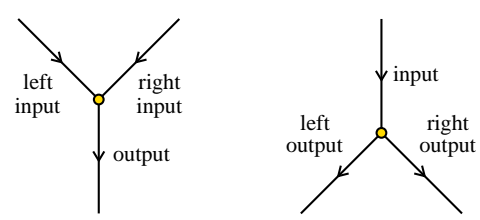

Fig. 1. A merge and a split (image taken from [7]).

$O(n)$ time. Given a linear time algorithm for determining isomorphism of planar graphs, as is theoretically possible according to Hopcroft and Wong [8], this gives rise to a linear time algorithm for the conjugacy problem in Thompson's Group $F$. The Hopcroft-Wong algorithm is quite complex, and no implementation of it currently exists. Therefore, we also describe a $O\left(n^{2}\right)$ Java program for checking conjugacy in $F$.

For an alternate approach to solving the conjugacy problem in $F$, the reader is referred to [9].

\section{AnNUlar STRAND Diagrams}

All of the material in this section are taken from [7].

$$
\begin{aligned}
& F=\left\langle x_{0}, x_{1} \mid x_{2} x_{1}=x_{1} x_{3}, x_{3} x_{1}=x_{1} x_{4}\right\rangle \\
& \text { where } x_{n} \text { is shorthand for } x_{0}^{1-n} x_{1} x_{0}^{n-1} \text { for } n \geq 2 .
\end{aligned}
$$

This group is well-known in geometric group theory, and has been studied extensively. See [4] for a comprehensive introduction to $F$.

Guba and Sapir [5], [6] provided a solution to the conjugacy problem in Thompson's group $F$ using graphs called diagrams. Building upon this solution, Belk and Matucci [7] introduced certain directed graphs called strand diagrams, and described a reduction of the conjugacy problem in group $F$ to isotopy of strand diagrams. In this paper we describe a further reduction of this problem to isomorphism of planar graphs and include an implementation of this reduction. This reduction algorithm takes as input two words in $\left\{x_{0}, x_{1}\right\}$ of length at most $n$, and produces two planar graphs in

Francesco Matucci gratefully acknowledges the Fondation Mathematique Jacques Hadamard (FMJH - ANR - Investissement d'Avenir for the support received during the development of this work.
Definition 1. A simple annular strand diagram is a finite directed multigraph drawn on an annulus without edge crossings, having the following properties:

1) Each vertex has degree three, and is either a merge or a split (see Fig. 1).

2) Every directed cycle winds counterclockwise around the central hole.

An annular strand diagram is a simple annular strand diagram along with a finite number of free loops, which are directed cycles without any vertices.

In [7], Belk and Matucci describe a procedure for obtaining an annular strand diagram corresponding to any element of $F$. Specifically, let $B\left(x_{0}\right), B\left(x_{1}\right), B\left(x_{0}^{-1}\right)$ and $B\left(x_{1}^{-1}\right)$ denote the four building blocks shown in Fig. 2. Given any word $s_{1} s_{2} \cdots s_{n}$ in $F$, i.e. any finite sequence of elements from $\left\{x_{0}, x_{0}^{-1}, x_{1}, x_{1}^{-1}\right\}$, the corresponding annular strand diagram is obtained by gluing together the building blocks $B\left(s_{1}\right), B\left(s_{2}\right), \ldots, B\left(s_{n}\right)$ in counterclockwise order around 


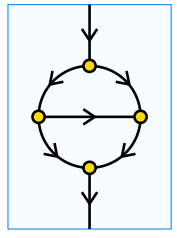

(a) $B\left(x_{0}\right)$

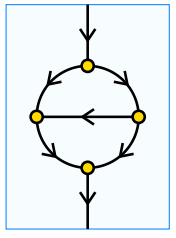

(b) $B\left(x_{0}^{-1}\right)$

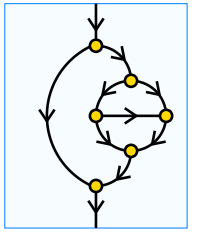

(c) $B\left(x_{1}\right)$

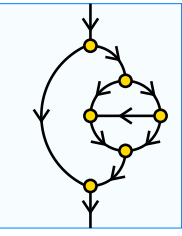

(d) $B\left(x_{1}^{-1}\right)$

Fig. 2. The four building blocks $B\left(x_{0}\right), B\left(x_{0}^{-1}\right), B\left(x_{1}\right), B\left(x_{1}^{-1}\right)$, corresponding to the generators for $F$ and their inverses.

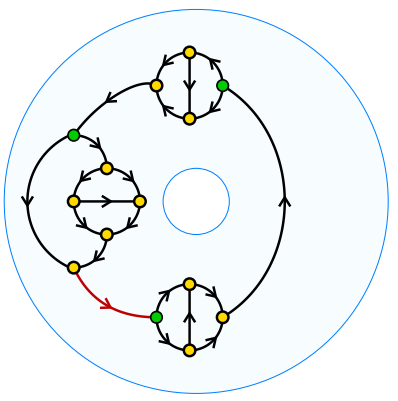

Fig. 3. Annular strand diagram corresponding to the word $x_{0} x_{0} x_{1}$. Each green split is marker for the start of its building block. The red edge glues the last building block to the first, creating a directed cycle.

the annulus, as shown in Fig. 3. Note that different words for the same element of $F$ will correspond to different annular strand diagrams.

Before stating the conjugacy theorem from [7], we need a few more definitions:

- A reduction of an annular strand diagram is a simplification of the directed graph using one of the three moves shown in Fig. 4. This set of reductions is confluent and terminating [7], so every annular strand diagram reduces to a unique normal form. We say that an annular strand diagram has been reduced if it is a normal form for this rewriting system. Fig. 5 shows the reductions performed on an annular strand diagram until it is reduced.

- Two annular strand diagrams are said to be isotopic if the second can be obtained from the first by some continuous motion in the annulus. The edges of the diagram are not

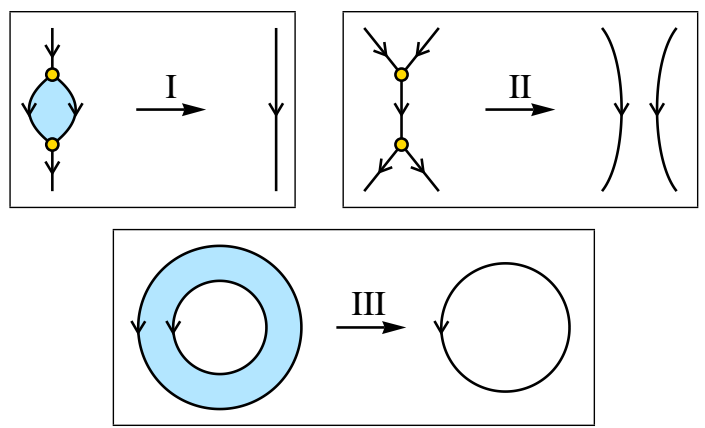

Fig. 4. The three reduction rules for annular strand diagrams. The blue indicates "empty regions", i.e. regions which are devoid of vertices and edges, and do not contain the central hole of the annulus.

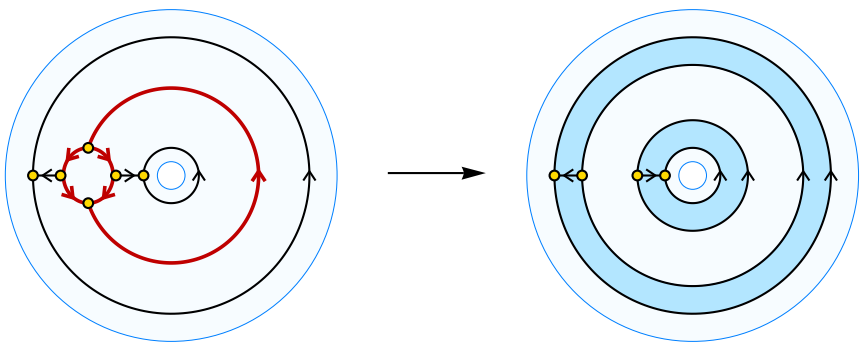

Fig. 6. A type II move at the red region splits the connected annular strand diagram into two connected components.

allowed to cross during this motion, and no edge may move across the central hole of the annulus.

We now present the solution to the conjugacy problem in $F$ described by Belk and Matucci [7].

Theorem 1. Let $a=a_{1} \cdots a_{m}$ and $b=b_{1} \cdots b_{n}$ be words representing elements of Thompson's group F. Let $A$ and $B$ be the corresponding annular strand diagrams, and let $A^{\prime}$ and $B^{\prime}$ be reduced annular strand diagrams obtained by reducing $A$ and $B$, respectively. Then $a$ and $b$ represent conjugate elements of $F$ if and only if $A^{\prime}$ and $B^{\prime}$ are isotopic.

Our algorithm simplifies annular strand diagrams through the rewrite rules of Fig. 4 in linear time before performing any check for isotopy of annular strand diagrams.

\section{CHECKING FOR ISOTOPY}

During the process of reduction, an annular strand diagram might become disconnected into several components. For example, Fig. 6 shows an annular strand diagram that splits into two components after a type II reduction. Keeping track of the relative positions of these components is one of the most difficult aspects of our algorithm.

Because every vertex of an annular strand diagram has at least one outgoing edge, every component must have at least one directed cycle. Since directed cycles are required to surround the central hole, it follows that the connected components of an annular strand diagram are arranged concentrically around the central hole of the annulus. We will use the following concept to keep track of the concentric order of these components.

Definition 2. Let $A$ be an annular strand diagram. A cutting path in $A$ is a path from the central hole of the annulus to the outside circle such that the cutting path crosses an edge only from left to right with respect to the orientation of the edge (see Fig. 7).

The following theorem explains the usefulness of cutting paths.

Theorem 2. Let $A_{1}, \ldots, A_{m}$ be the connected components of a reduced annular strand diagram $A^{\prime}$ in concentric order, and let $e_{1}, \ldots, e_{n}$ be the sequence of edges crossed by a cutting path 

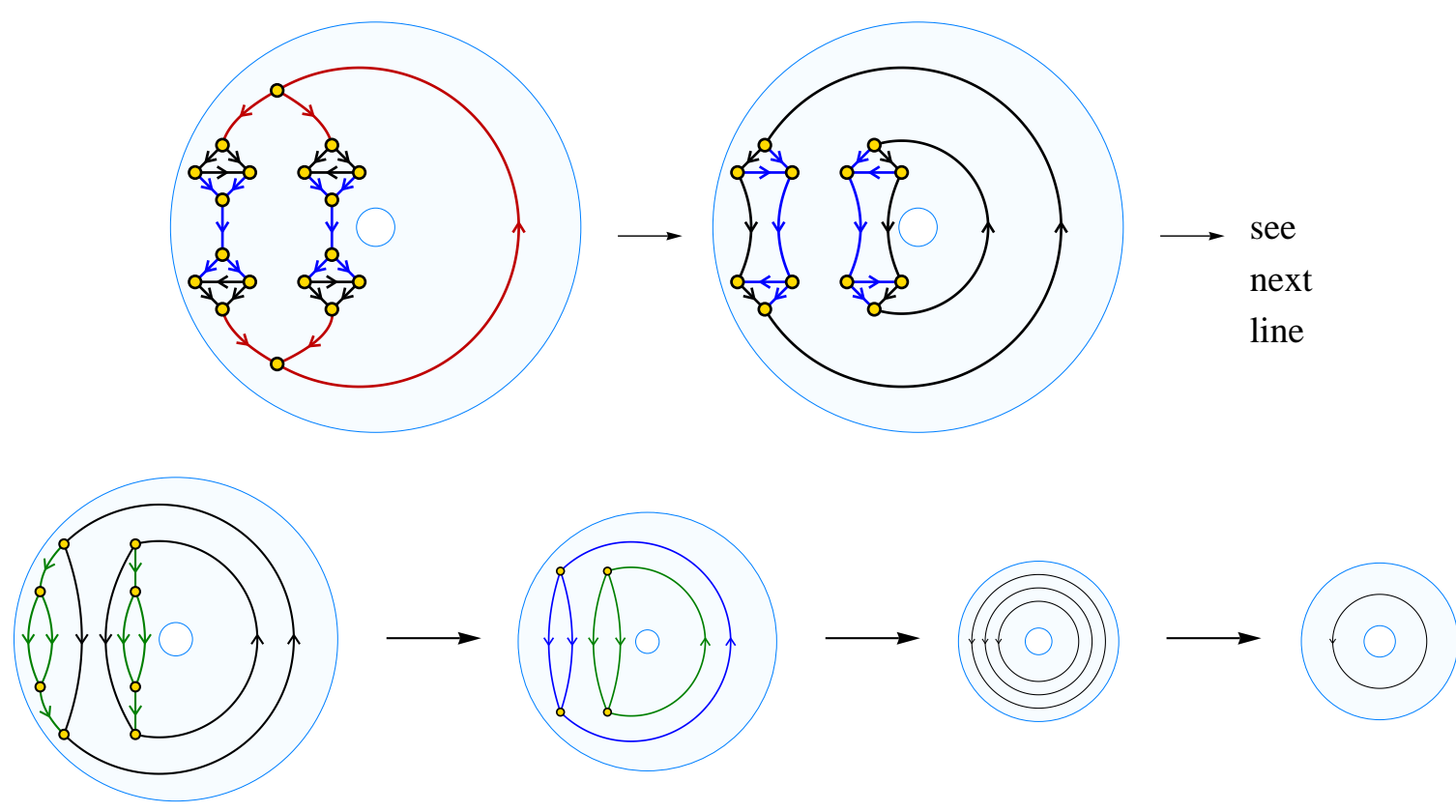

Fig. 5. Reducing an annular strand diagram to a free loop. The green regions are subject to type I moves, and the red and blue regions are each subject to type II moves.

for $A^{\prime}$. Then $e_{1}, \ldots, e_{n}$ consists of one or more edges from $A_{1}$ followed by one or more edges from $A_{2}$ and so forth, ending with one or more edges from $A_{m}$.

To prove this theorem, we require the following lemma on the structure of reduced annular strand diagrams.

Lemma 1. Let $A^{\prime}$ be a reduced annular strand diagram. Then each component of $A^{\prime}$ that is not a free loop lies in a closed annular region bounded by two directed cycles.

Proof: Let $A_{i}$ be a component of $A^{\prime}$ that is not a free loop, and consider any directed cycle of $A_{i}$. If this cycle were to have both merges and splits, then at some point there would be a merge followed by a split, which would be subject to a type II reduction. Since $A^{\prime}$ is reduced, it follows that every directed cycle in $A_{i}$ consists solely of either merges or splits. Therefore, all of the edges attached to the outermost directed cycle of $A_{i}$ must lie on the inside of the cycle, and all of the edges attached to the innermost cycle of $A_{i}$ must lie on the outside of the cycle, so $A_{i}$ lies in the annular region between these two cycles.
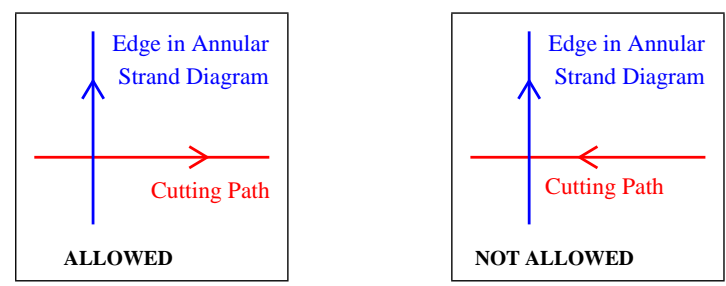

Fig. 7. The cutting path crosses each edge in the annular strand diagram from left to right.
Proof of Theorem 2: Clearly the cutting path must cross each of the components $A_{i}$ at least once. Moreover, because of the edge crossing rules for cutting paths, a cutting path can cross a directed cycle only one time. Since every component is either a free loop or is bounded by two directed cycles, the result follows.

Our algorithm keeps track of a cutting path for the annular strand diagram of each element, modifying the path as the annular strand diagram is reduced. After reduction, we use Theorem 2 to reconstruct the concentric order of the components, a necessary step in checking for isotopy. Indeed, because of the concentric arrangement of the components, we can check isotopy for each pair of components separately:

Proposition 1. Let $A$ be an annular strand diagram with components $A_{1}, \ldots, A_{m}$ in concentric order, and let $B$ be an annular strand diagram with components $B_{1}, \ldots, B_{n}$ in concentric order. Then $A$ and $B$ are isotopic if and only if $m=n$ and $A_{i}$ is isotopic to $B_{i}$ for each $i$.

We use the following theorem to check isotopy for connected components:

Theorem 3. Let $A_{i}$ and $B_{i}$ be connected annular strand diagrams. Then $A_{i}$ and $B_{i}$ are isotopic if and only if there exists an isomorphism $\varphi: A_{i} \rightarrow B_{i}$ of directed multigraphs satisfying the following conditions:

1) For every split vertex $v$ in $A_{i}$, the isomorphism $\varphi$ maps the left output of $v$ to the left output of $\varphi(v)$, and the right output of $v$ to the right output of $\varphi(v)$.

2) For every merge vertex $v$ in $A_{i}$, the isomorphism $\varphi$ maps the left input of $v$ to the left input of $\varphi(v)$, and the right 
input of $v$ to the right input of $\varphi(v)$.

Proof: Observe that an isomorphism $\varphi$ satisfies conditions (1) and (2) if and only if it preserves the counterclockwise order of the edges incident on each vertex. That is, $\varphi$ satisfies (1) and (2) if and only if $\varphi$ respects the "rotation systems" associated to $A_{i}$ and $B_{i}$ (see [10]). Therefore, there exists an isomorphism $\varphi$ satisfying (1) and (2) if and only if $A_{i}$ and $B_{i}$ are isotopic as directed graphs on a sphere.

To relate isotopy on the sphere with isotopy on the annulus, observe that the region of $A_{i}$ containing the central hole is the only region whose boundary is a counterclockwise directed cycle. Similarly, the region of $A_{i}$ corresponding to the outside of the annulus is the only region whose boundary is a clockwise directed cycle. The same holds true for $B_{i}$. Therefore, given any isotopy from $A_{i}$ to $B_{i}$ on the sphere, the regions containing the central holes must correspond, as must the outer regions. It follows that $A_{i}$ and $B_{i}$ are isotopic on the sphere if and only if they are isotopic on the annulus.

\section{THE AlgorithM}

In this section, we describe and analyze the algorithm for the conjugacy problem in $F$. This algorithm refines the solution stated in Theorem 1 to achieve the best possible running time.

The steps in the algorithm are summarized in Fig. 8. We believe that the following key points will make it easier for the reader to understand the analysis of the algorithm presented later in this section:

1) The two inputs $w 1$ and $w 2$ are strings in the alphabet $\left\{x_{0}, x_{1}, x_{0}^{-1}, x_{1}^{-1}\right\}$.

2) The algorithm keeps track of a cutting path. After the annular strand diagrams are reduced, their connected components are labeled in concentric order using the sequence of edges in this cutting path (see Theorem 2).

3) We reduce the problem of determining whether two reduced annular strand diagrams are isotopic to the problem of determining whether two planar graphs are

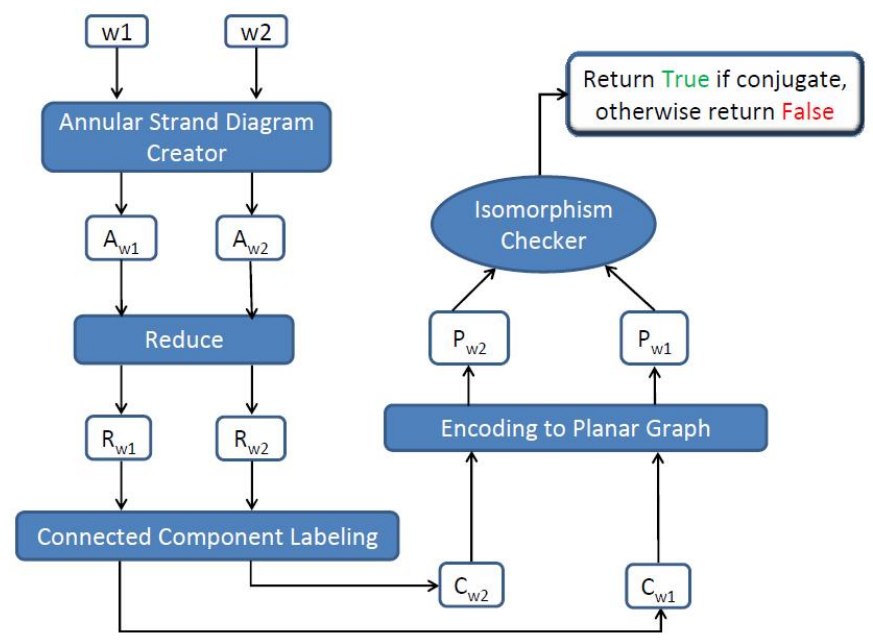

Fig. 8. Overview of the algorithm for the conjugacy problem in Group $F$ isomorphic. This is done by applying a one-to-one encoding on each connected component to convert it to a connected planar graph. The purpose of this step is to apply the $O(|V|)$ algorithm proposed by Hopcroft and Wong [8] for the isomorphism problem in planar graphs to make our algorithm achieve a linear running time. Whether $w 1$ and $w 2$ represent conjugate elements is then decided by using Proposition 1.

Theorem 4. Given two input words $w_{1}$ and $w_{2}$ in $\left\langle x_{0}, x_{1}\right\rangle$ representing two elements of $F$, the proposed algorithm for the conjugacy problem decides whether the two elements are conjugate in $O(n)$, where $n=\left|w_{1}\right|+\left|w_{2}\right|$.

The rest of this section proves this theorem.

\section{A. The Data Structure}

Table I shows the data structure for representation and manipulation of annular strand diagrams.

Below we discuss the data structure, with particular emphasis on key fields and methods.

Class: Edge

This class holds edges in annular strand diagrams.

1) The field type is an array of two integers that records the type to which the edge belongs (see Section IV-D for a discussion of "type"). In this array, the first integer denotes the input type and the second integer denotes the output type for the edge. These integers can be the following:

- $0 \rightarrow$ free loop

- $1 \rightarrow$ left input or left output

- $2 \rightarrow$ right input or right output

2) The node field denotes the container node in the linked list representing the cutting path to which the edge belongs.

3) Given an edge $e_{1}$ with source vertex $s$, the combineEdge () method takes an edge $e_{2}$ with target vertex $t$ as input, and then merges the two edges. As a result, both $e_{1}$ and $e_{2}$ are the same edge with source vertex $s$, target vertex $t$, and their type and node fields are modified if required.

\section{Class: Vertex}

The Vertex class represents merges and splits.

The field type denotes the vertex type, which can be either "merge" or "split". Using the type field, we can safely decide which of the four Edge fields are valid for a vertex, as shown in Table II.

TABLE II

THE EDGE OBJECTS ASSOCIATED WITH CERTAIN VERTEX TYPES.

\begin{tabular}{c|c|c} 
& merge & split \\
\hline leftParentEdge & $\checkmark$ & $\checkmark$ \\
\hline rightParentEdge & $\checkmark$ & $\boldsymbol{X}$ \\
\hline leftChildEdge & $\checkmark$ & $\checkmark$ \\
\hline rightChildEdge & $\boldsymbol{X}$ & $\checkmark$
\end{tabular}


TABLE I

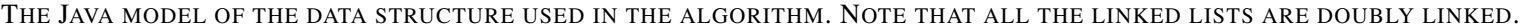

\begin{tabular}{|c|c|c|c|c|}
\hline Class & Vertex & Edge & Annular & Graph \\
\hline Fields & $\begin{array}{l}\text { type: String } \\
\text { leftParentEdge: Edge } \\
\text { rightParentEdge: Edge } \\
\text { leftChildEdge: Edge } \\
\text { rightChildEdge: Edge } \\
\text { ID: Integer } \\
\text { node: Node }<\text { Vertex> } \\
\text { isPaired: Boolean } \\
\text { correspondent: Vertex }\end{array}$ & $\begin{array}{l}\text { source: Vertex } \\
\text { target: Vertex } \\
\text { ID: Integer } \\
\text { type: Integer } \\
\text { isFreeLoop: Boolean } \\
\text { node: Node<Edge> } \\
\text { flagged: Boolean }\end{array}$ & $\begin{array}{l}\text { vertices: LinkedList }<\text { Vertices }> \\
\text { stackReduceSplits: Stack }<\text { Vertex }> \\
\text { cuttingPath: LinkedList }<\text { Edge }>\end{array}$ & $\begin{array}{l}\text { vertices: List }<\text { Vertex }> \\
\text { edges: List }<\text { Edge }>\end{array}$ \\
\hline Methods & $\begin{array}{l}\text { getLeftParent(): Vertex } \\
\text { getRightParent(): Vertex } \\
\text { getLeftChild(): Vertex } \\
\text { getRightChild(): Vertex }\end{array}$ & $\begin{array}{l}\text { combineEdge(Edge) } \\
\text { makeFreeLoop() }\end{array}$ & $\begin{array}{l}\text { reduce() } \\
\text { getComponents() } \\
\text { encodeToPlanarGraph() }\end{array}$ & \\
\hline
\end{tabular}

Note that the Vertex data structure keeps track of the counterclockwise order of the edges (i.e. the rotation system) since it keeps track of the left and right parents of a merge, and similarly the left and the right children of a split. Therefore, by Theorem 3, this data structure is sufficient to keep track of the isotopy class of the annular strand diagram.

Class: Graph

The Graph data structure is used to hold the planar graphs that are generated from the components of reduced annular strand diagram (discussed in Section IV-D). A list of the vertices and a list of the undirected edges are sufficient to represent these planar graphs.

Class: Annular

This data structure represents elements of $F$ in annular strand diagram forms.

1) An Annular object is constructed by going through the input word from left to right, and creating and gluing the corresponding building blocks together.

2) The stack stackReduceSplits stores vertices that may be involved in reduction (discussed in Sections IV-B and IV-C).

3) The field cuttingPath is a linked list that stores a sequence of edges in a particular cutting path in the annular strand diagram.

4) The reduce () method performs all the possible reduction moves on an annular strand diagram, thereby reducing it.

5) The getComponents () method returns a concentric ordered list of the connected components in the annular strand diagram. These connected components are also Annular objects.

6) The method encodeToPlanarGraph() encodes connected components to planar graphs, which are Graph objects.

Now we begin a thorough discussion and analysis of the algorithm for the conjugacy problem.

\section{B. Annular Strand Diagram Generation}

Each building block for creating an annular strand diagram has a constant number of vertices and edges (see Fig. 2).
Therefore, construction of the annular strand diagram corresponding to the input word requires $O(n)$ vertices and edges, and $O(n)$ gluing of the building blocks, proving that creation of an annular strand diagram is $O(n)$. Note the following key points:

1) During the construction of an annular strand diagram, we put all the splits at the gluing points into a stack called stackReduceSplits as we know that these splits mark the regions of all possible reductions that can be performed on the annular strand diagram at that instant. To be precise, these regions are exposed to type II reduction moves. For instance, in Fig. 3, these are the green splits.

2) The edge that glues the last building block to the first is added to a doubly linked list called cuttingPath that represents the cutting path which the algorithm will dynamically keep track of.

\section{Reduce}

We now analyze the reduce () method and prove that it takes $O(n)$ to reduce an annular strand diagram. For our purposes, it suffices to show that the cutting path update, the number of reductions, and the number of checks for reductions collectively take $O(n)$.

Cutting Path Update: Fig. 9 shows the strategy we employ to update the cutting path. Reductions are performed by first removing edges and vertices, and then combining edges using combineEdge (). Each new edge created after a reduction represents both the edges that were combined to create the new edge. This means that in the case of a reduction I, we do not need to worry about updating the cutting path if it crosses edges $e_{1}$ or $e_{4}$ prior to the reduction because the reduction will update the cutting path accordingly. Similarly, in the case of a reduction II, we do not need to update the cutting path if it does not cross $e_{3}$ prior to the reduction. Hence, the only cases where the algorithm has to update the cutting path are the cases shown in Fig. 9. Also recall that the node field for edges ensures that each edge knows whether it is in the cutting path. Therefore, it is easy to see that deciding whether the cutting path needs to be updated during a reduction, and also updating the cutting path during a reduction both take 


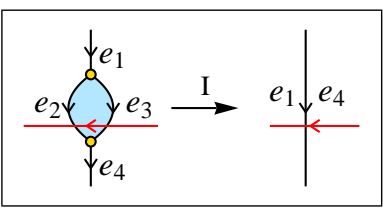

(a) reduction $\mathrm{I}$

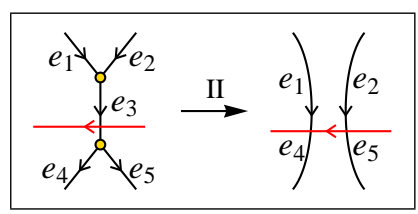

(b) reduction II

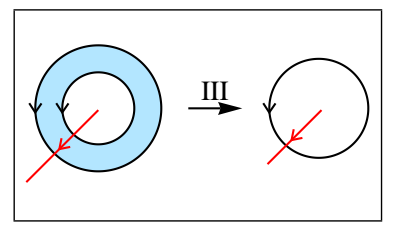

(c) reduction III

Fig. 9. Update of the cutting path (colored red) for each reduction move.

$O(1)$. Fig. 10 shows the edges in an annular strand diagram that the cutting path intersects before and after the annular strand diagram is reduced.

Number of Reductions: Observe that each type I or type II move deletes two vertices from an annular strand diagram. Since the number of vertices in the annular strand diagram after its creation is $O(n)$, it follows that the number of type I or type II moves is also $O(n)$. The number of type III moves is also bounded by $O(n)$ because each of these moves merge two concentric edges, and there can be at most $O(n)$ concentric edges in the annular strand diagram.

Number of Checks for Reductions: Observe that a reduction can create a new region of reduction nearby. We perform reductions locally and keep track of all possible regions in which new reductions may appear as a result of a local reduction. Notice that both type I or type II moves happen around a split. We use stackReduceSplits to store all possible splits that may be involved in these reduction moves. After any of these reduction moves are performed, we push onto stackReduceSplits the splits connected to the newly created edges (for instance, the splits connected to $e_{1}$ in Fig. 9.(a) after the reduction) because such a split might be now exposed to a reduction move. In this way we can check for all possible type I and type II moves. Observe that these reductions involve a constant number of pushes onto the stack. Because there are $O(n)$ reductions, there are at most $O(n)$ pushes onto the stack, and hence $O(n)$ checks for reduction I and II. We perform the type III moves after all possible type I and type II moves are performed. The type III moves are detected by finding all the adjacent free loops in the cutting path, which involve $O(n)$ checks since the cutting path can have at most $O(n)$ edges in it. This proves that the reduce () method takes $O(n)$.

After the annular strand diagrams are reduced, the connected components are detected in concentric order.

Connected Component Labeling: Recall that the data structure for edges holds the source and target vertices, and the data structure for vertices holds the connected edges. Therefore, given the cutting path for a reduced annular strand diagram, for each edge that meets the cutting path in order, we can perform a breadth first search along both directions of the edge to discover the connected component to which the edge belongs. Because all the components collectively have a sum of vertices and edges bounded by $O(n)$, it follows that all connected components are identified in concentric order in $O(n)$.

\section{Encoding to Planar Graphs}

In this section, we explain and analyze the part of the algorithm which reduces the problem of determining isotopic annular strand diagrams to the problem of determining isomorphic planar graphs.

Theorem 5. Any two connected, reduced annular strand diagrams $A_{1}^{\prime}$ and $A_{2}^{\prime}$ can be encoded into two planar graphs $p_{1}$ and $p_{2}$ respectively such that $A_{1}^{\prime}$ and $A_{2}^{\prime}$ are isotopic if and only if $p_{1}$ and $p_{2}$ are isomorphic.

Sketch of Proof: To prove the theorem above, it suffices to demonstrate an encoding that is one-to-one. First we will describe the encoding, and then show that it is one-to-one.

We will now describe the function $\phi$ that encodes connected, reduced annular strand diagrams into planar graphs. First, notice that there are three possible input types for an edge, namely the left input or the right input to a merge, or the lone input to a split (see Fig. 1). Similarly an edge can have three possible output types. Hence there are nine different inputoutput combinations for an edge. In addition, an edge can be a free loop. Therefore, we have ten different types of edges. We provide unique encodings of each type of edge, as shown in Table III that $\phi$ will make use of. Note that the type to which an edge $e$ belongs uniquely identifies the corresponding planar graph for the edge using the number of edges created between the vertices $u$ and $v_{2}$ in the corresponding planar graph (see Table III).

Let $\phi: X \rightarrow G$ be a function that maps the set of connected, reduced annular strand diagrams $X$ to the set of planar graphs $G$. Assume that $E=\left\{e_{1}, e_{2}, \ldots e_{n}\right\}$ is the edge set of $A^{\prime} \in X$. To obtain $p_{A^{\prime}}=\phi\left(A^{\prime}\right)$, follow the steps below:

1) Create a null graph $p_{A^{\prime}}$.

2) Copy all the vertices from $A^{\prime}$ to $p_{A^{\prime}}$.

3) For each edge $e \in E$, identify its edge type in Table III, and perform the appropriate encoding of $e$.

The function $\phi$ uniquely encodes its input annular strand diagram to a planar graph. Moreover, this process is one-toone. Indeed if two (reduced) annular strand diagrams encode the same planar graph via $\phi$, then it is easy to recover the original vertices and determine the left and right inputs of corresponding merges, and the left and right outputs of corresponding splits. This means that both annular strand diagrams have the same rotation systems. Theorem 3 asserts that they must be isotopic.

Analysis of the Encoding Algorithm: Recall that all the connected components together have a total of $O(n)$ vertices and edges. Because the encoding creates a constant number of vertices and edges for each edge in $E$, it follows that $\phi$ creates a planar graph with $O(n)$ vertices and edges. 


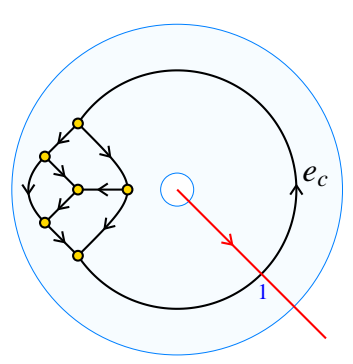

(a)
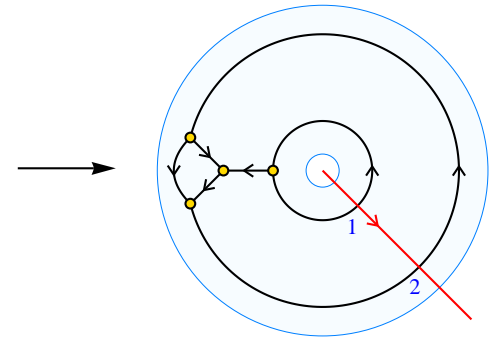

(b)

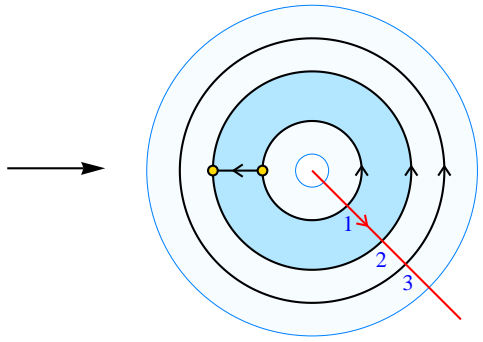

(c)

Fig. 10. Status of a cutting path (a) after closing a strand diagram (crosses edge $e_{c}$ ), (b) after performing a type II reduction, and (c) after the annular strand diagram is reduced. The numbers denote the order in which the cutting path crosses the edges of the annular strand diagram.

\section{E. Determining Isotopy}

To check whether two reduced annular strand diagrams are isotopic, we use Proposition 1 and Theorem 5. In other words, all corresponding planar graphs from both annular strand diagrams are compared to determine whether they are isomorphic using the $O(V)$ algorithm for the isomorphism problem in planar graphs proposed by Hopcroft and Wong [8], where $V$ is the number of vertices in the input. Note that their algorithm accepts planar graphs with loops and multiple edges between vertices. Because the total number of vertices from our planar graph encodings are collectively bounded by $O(n)$, it follows that all the checks for isomorphism between planar graphs take $O(n)$.

This concludes the proof of Theorem 4, confirming that the algorithm for the conjugacy problem is linear in the input size. A Java-style pseudocode version of the algorithm is shown in Algorithm 1.

\section{F. Implementation}

To the best of our knowledge, the linear time algorithm for the isomorphism problem in planar graphs proposed in [8] has not been implemented yet. This is due to the complicated design of the algorithm. Moreover, the authors of [8] stated that this algorithm is not practical. As a result, we have not attempted an implementation of this algorithm, and instead programmed a direct isotopy search as a substitute for the following steps:

1) planar graph encoding of each component in the two annular strand diagrams, and

2) checking for isomorphism between all corresponding pairs of planar graph encodings.

Our implementation, called ConjugacyF, is a brute force implementation of Theorem 3. In particular, it substitutes the two steps above with a method that checks the rotation systems of the two reduced annular strand diagrams to determine whether the graphs are isotopic. This involves fixing a reference vertex $v_{r}$ in one of the reduced annular strand diagrams $A_{1}^{\prime}$, and then for each vertex $v_{c}$ in the other reduced annular strand diagram $A_{2}^{\prime}$ as a possible correspondent of $v_{r}$, a breadth first expansion is performed in both $A_{1}^{\prime}$ and $A_{2}^{\prime}$ along the edges connected to each of $v_{r}$ and $v_{c}$ in counterclockwise order. If any of these expansions produces the same graphs, then $A_{1}^{\prime}$

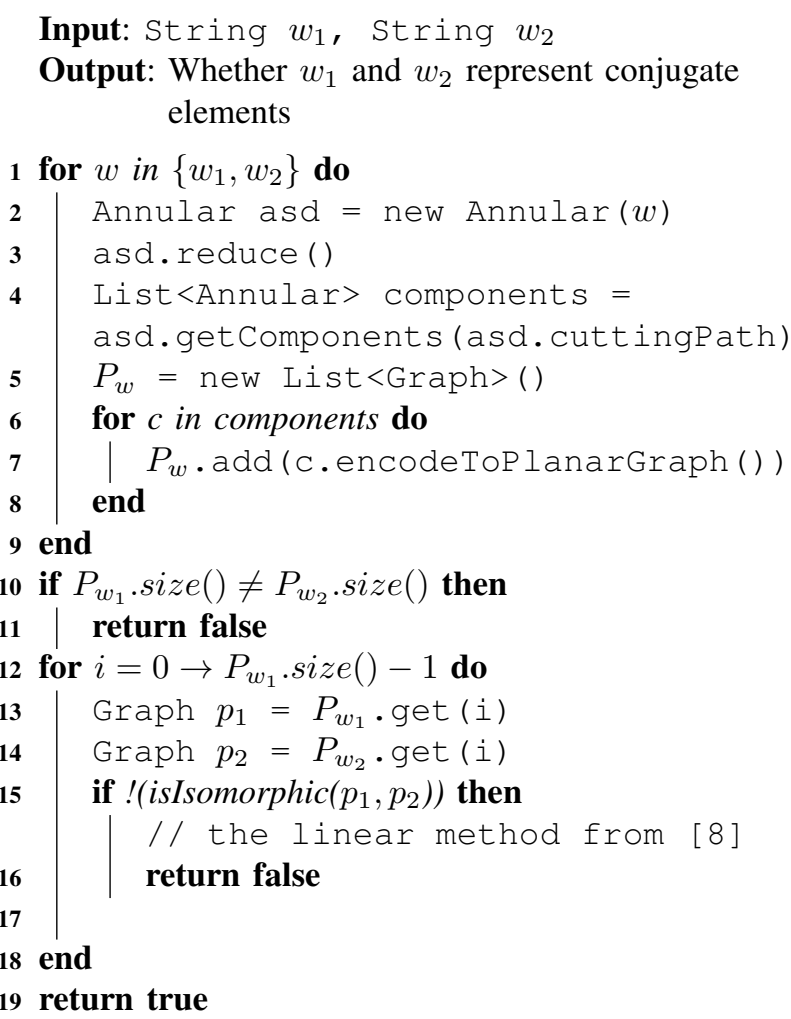

Algorithm 1: Algorithm for the conjugacy problem in $F$.

and $A_{2}^{\prime}$ represent conjugate elements. In the worst case (when the graphs are not isotopic), this algorithm performs two linear time expansions for each vertex $v_{c}$ in $A_{2}^{\prime}$. Hence ConjugacyF is $O\left(n^{2}\right)$.

We made ConjugacyF publicly available on [11] as a web application in the form of a Java applet and an executable JAR file (compiled on Windows 7). These applications allow users to check whether two elements of $F$ are conjugate, to sort a list of elements into conjugacy classes, and to compute the reduced annular strand diagram for any element. We also shared the Java source code for ConjugacyF on [11]. 
TABLE III

DIFFERENT TYPES OF EDGES IN THE ANNULAR STRAND DIAGRAM, AND THEIR ENCODINGS TO PLANAR GRAPHS
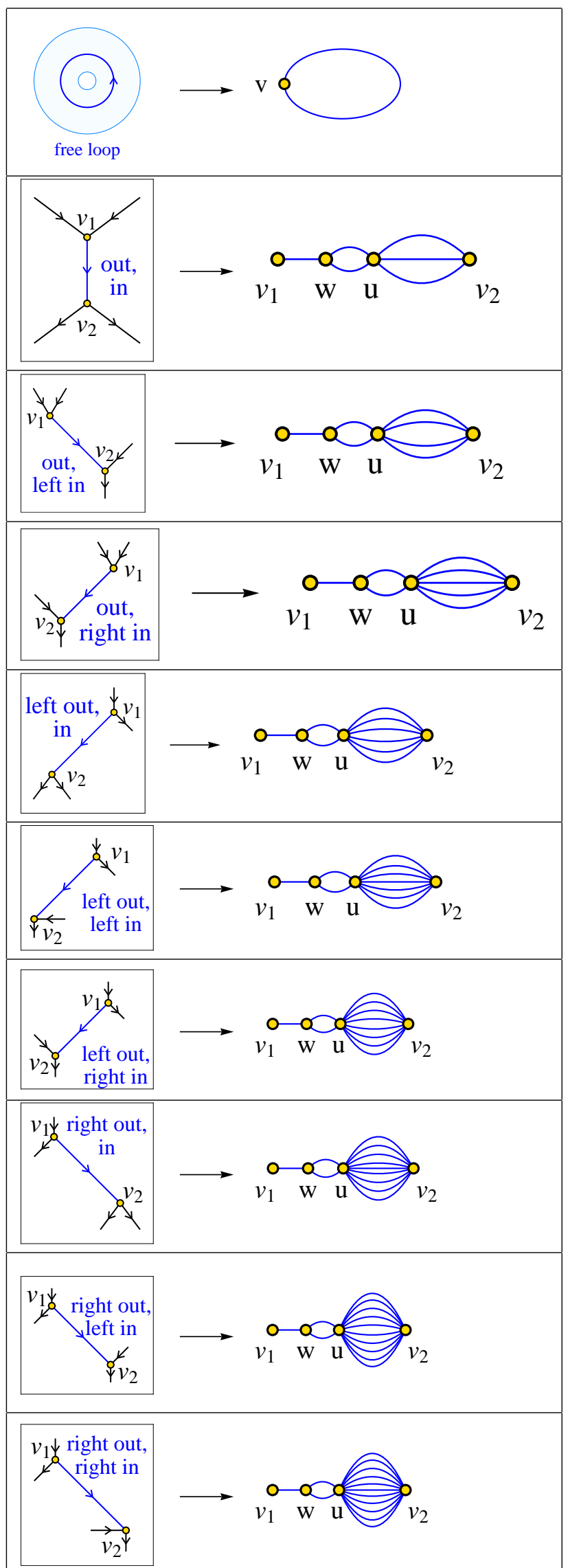

\section{CONCLUSion ANd Future Work}

We presented a linear time reduction of the conjugacy problem in Thompson's Group $F$ to the isomorphism problem of planar graphs using directed graphs called annular strand diagrams, along with data structures for efficient storage and manipulation of the associated mathematical objects. Given a linear time algorithm for the isomorphism problem of planar graphs [8], this leads to a linear time algorithm for the conjugacy problem in $F$. Moreover, the conjugacy problem in $F$ requires linear time, so such an algorithm represents the best runtime generally speaking.

Due to the impractical nature of the linear algorithm for the isomorphism problem in planar graphs [8], we implemented a quadratic solution that directly determines whether two reduced annular strand diagrams are isotopic, and we made the software publicly available. This is the first public software implementation of an algorithm for the conjugacy problem in $F$. It is our hope that our software will be useful to the research community in Thompson's Groups.

For future work, we believe that it would not be too hard to modify our software to create algorithms for the conjugacy problems in the other two Thompson's Groups, namely $V$ and $T$. The cutting path used in the algorithm for $F$ is representative of the cutting class [7] used in solving the conjugacy problem in $V$. However, the algorithm for $V$ is not expected to be linear because checking whether two cutting paths represent the same cutting class might require Gaussian elimination [12], which is worse than linear.

\section{REFERENCES}

[1] P. S. Novikov, "Unsolvability of the conjugacy problem in the theory of groups.(Russian)," Izv. Akad. Nauk SSSR. Ser. Mat, vol. 18, pp. 485-524, 1954.

[2] K. Madlener and J. Avenhaus, "String matching and algorithmic problems in free groups." Revista colombiana de matematicas, vol. 14, pp. $1-16,1980$.

[3] F. A. Garside, "The braid group and other groups," The Quarterly Journal of Mathematics, vol. 20, no. 1, pp. 235-254, 1969.

[4] J. W. Canon, W. J. Floyd, and W. R. Parry, "Introductory notes on Richard Thompson's groups," Enseignement Mathématique, vol. 42, pp. 215-256, 1996.

[5] V. Guba and M. V. Sapir, Diagram groups. AMS Bookstore, 1997, vol. 620 .

[6] V. S. Guba and M. V. Sapir, "On subgroups of R. Thompson's group F and other diagram groups," Sbornik: Mathematics, vol. 190, no. 8, p. 1077, 1999.

[7] J. Belk and F. Matucci, "Conjugacy and dynamics in Thompson's groups," Geometriae Dedicata, pp. 1-23, 2013. [Online]. Available: http://dx.doi.org/10.1007/s10711-013-9853-2

[8] J. E. Hopcroft and J.-K. Wong, "Linear time algorithm for isomorphism of planar graphs (preliminary report)," in Proceedings of the sixth annual ACM symposium on Theory of computing. ACM, 1974, pp. 172-184.

[9] I. Short and N. Gill, "Conjugacy in Thompson's group F," Proceedings of the American Mathematical Society, vol. 141, pp. 1529-1538, 2013.

[10] J. L. Gross and S. R. Alpert, "The topological theory of current graphs," Journal of Combinatorial Theory, Series B, vol. 17, no. 3, pp. 218-233, 1974.

[11] N. T. Hossain. (2013, May) Algorithm for the conjugacy problem in Thompson's group F. Accessed: 30 June 2013. [Online]. Available: http://www.asclab.org/asc/nhossain/conjugacyF

[12] J. Edmonds, "Systems of distinct representatives and linear algebra," $J$. Res. Nat. Bur. Standards, Sect. B, vol. 71, no. 4, pp. 241-245, 1967. 